\title{
Seismic glitchness at Sos Enattos site: impact on intermediate black hole binaries detection efficiency
}

\author{
A. Allocca ${ }^{1,2}$, A. Berbellini ${ }^{3}$, L. Boschi ${ }^{3,4,5}$, E. Calloni ${ }^{1,2, a}$ (D) G. L. Cardello ${ }^{6,7}$,

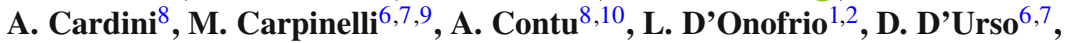 \\ D. Dell'Aquila ${ }^{6,7}$, R. De Rosa ${ }^{1,2}$, L. Di Fiore ${ }^{2}$, M. Di Giovanni ${ }^{11,12,13}$, S. Di Pace ${ }^{14,15}$, \\ L. Errico ${ }^{1,2}$, I. Fiori ${ }^{9}$, C. Giunchi ${ }^{11}$, A. Grado ${ }^{16}$, J. Harms ${ }^{12}$, E. Majorana ${ }^{14,15}$, \\ V. Mangano ${ }^{14,15}$, M Marsella ${ }^{14,15}$, C. Migoni $^{8}$, L. Naticchioni ${ }^{14,15}$, M. Olivieri ${ }^{3}$, \\ G. Oggiano $^{6,7}$, F. Paoletti ${ }^{17}$, M. Punturo ${ }^{18}$, P. Puppo ${ }^{15}$, P. Rapagnani ${ }^{14,15}$,

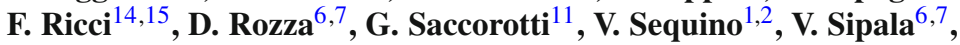 \\ I. Tosta E Melo ${ }^{6,7}$, L. Trozzo ${ }^{2}$ \\ ${ }^{1}$ Universitá Federico II Napoli, 80126 Napoli, Italy \\ 2 INFN - sezione di Napoli, 80126 Napoli, Italy \\ ${ }^{3}$ INGV - sezione di Bologna, 40128 Bologna, Italy \\ ${ }^{4}$ Universitá degli studi di Padova, 35131 Padova, Italy \\ ${ }^{5}$ Sorbonne Université, 75005 Paris, France \\ ${ }^{6}$ Universitá degli Studi di Sassari, 07100 Sassari, Italy \\ ${ }^{7}$ INFN - Laboratori Nazionali del Sud, 95125 Catania, Italy \\ ${ }^{8}$ INFN - sezione di Cagliari, 09042 Monserrato, CA, Italy \\ ${ }^{9}$ European Gravitational Observatory, 56012 Cascina (PI), Italy \\ ${ }^{10}$ INAF - Osservatorio Astronomico di Cagliari, 09042 Cagliarii, Italy \\ ${ }^{11}$ INGV - sezione di Pisa, 56123 Pisa, Italy \\ 12 Gran Sasso Science Institute, 67100 L'Aquila, Italy \\ 13 INFN - Laboratori Nazionali del Gran Sasso, 67100 Assergi, AQ, Italy \\ 14 Universitá di Roma La Sapienza, 00185 Roma, Italy \\ 15 INFN - sezione di Roma 1, 00185 Roma, Italy \\ 16 INAF - Osservatorio Astronomico di Capodimonte, 80131 Napoli, Italy \\ 17 INFN - sezione di Pisa, 56127 Pisa, Italy \\ 18 INFN - sezione di Perugia 1, 06123 Perugia, Italy
}

Received: 5 March 2021 / Accepted: 16 April 2021

(C) The Author(s) 2021

\begin{abstract}
Third-generation gravitational wave observatories will extend the lower frequency limit of the observation band toward $2 \mathrm{~Hz}$, where new sources of gravitational waves, in particular intermediate-mass black holes (IMBH), will be detected. In this frequency region, seismic noise will play an important role, mainly through the so-called Newtonian noise, i.e., the gravity-mediated coupling between ground motion and test mass displacements. The signal lifetime of such sources in the detector is of the order of tens of seconds. In order to determine whether a candidate site to host the Einstein Telescope observatory is particularly suitable to observe such sources, it is necessary to estimate the probability distributions that, in the characteristic time scale of the signal, the sensitivity of the detector is not perturbed by Newtonian noise. In this paper, a first analysis is presented, focused on the Sos Enattos site (Sardinia, Italy), a candidate to host the Einstein Telescope. Starting from a long data
\end{abstract}

\footnotetext{
a e-mail: enrico.calloni@na.infn.it (corresponding author)
} 
set of seismic noise, this distribution is evaluated considering both the presently designed triangular ET configuration and also the classical "L" configuration.

\section{Introduction}

The recent discovery of gravitational waves [1-3] has prompted the scientific community toward the realization of the so-called third-generation gravitational wave detectors. Among the most important technical improvements, there are the extension of the observation bandwidth at low frequency and the gain of about a factor ten in sensitivity in the bandwidth covered by present detectors $[4,5]$.

In particular, the Einstein Telescope (ET) [5] will be an underground, cryogenic detector, composed by three pairs of Michelson interferometers arranged in an equilateral triangle configuration, as shown in Fig. 1; each pair composes a xylophone, being constituted by an interferometer optimized for low frequencies and an interferometer optimized for high frequencies.

The low-frequency limit of the bandwidth will be extended from the present $10 \mathrm{~Hz}$ down to $2 \mathrm{~Hz}$. The study of several interesting sources of gravitational waves is expected to benefit from the extension of the bandwidth: rotating pulsars, coalescing binaries, and, of course, possible yet unknown sources [6]. Among possible new sources, a remarkable interest is given by Intermediate Mass Black Hole Binaries: coalescing binary systems with total mass ranging from about few hundreds solar masses up to several thousands and more [7].

Evidence of the existence of such sources was obtained from the observation of the GW190521 event: the mass of the initial black holes was 85 and 64 solar masses, and the final mass of the merger (142 solar masses) classifies it as an intermediate-mass black hole $[8,9]$.

The duration of these signals within the new observational window depends on several parameters. Indeed, depending on mass asymmetry, spin and distance, the duration of such signals in the detection bandwidth can vary from several tens of seconds to a few minutes.

Considering that a number of non-stationary noise can affect the detector $[10,11]$, it is important to investigate the behavior of such noises in the same time scale of the signal. Among the noises that are expected to mostly affect the detector in that bandwidth, the seismic noise has a key role. Indeed it can couple with the interferometer both through indirect ways, like for example by modulating the diffused light [12], or also in a direct way, through the variation of gravitational field due to displacements of ground masses nearby the interferometer test masses.

The latter is known as Newtonian noise [13], and the ET requirements are so stringent that the interferometer will be positioned underground, at a depth of about $250 \mathrm{~m}$, where the seismic noise is strongly reduced compared to the surface. Even in this condition, the Newtonian noise $(\mathrm{NN})$ remains one of the most critical noise sources, affecting in particular the bandwidth where the new detectors should extend. [13].

In the evaluation of the sites that could host such a third-generation detector, a first estimation of the residual noise has been obtained by evaluating the power spectral density of the seismic noise, during a long data-taking, to assess its median value. Presently two sites remain the candidate to host ET: the Dutch site Terziet, near Maastricht, at the border of Belgium, the Netherlands and Germany [14] and the Italian Sos Enattos (Lula - NU, in Sardinia) $[5,15,16]$.

In view of the detection of transients of the order of one minute, it is nevertheless important also to assess the distribution of the seismic noise over this time scale to evaluate, for example, 


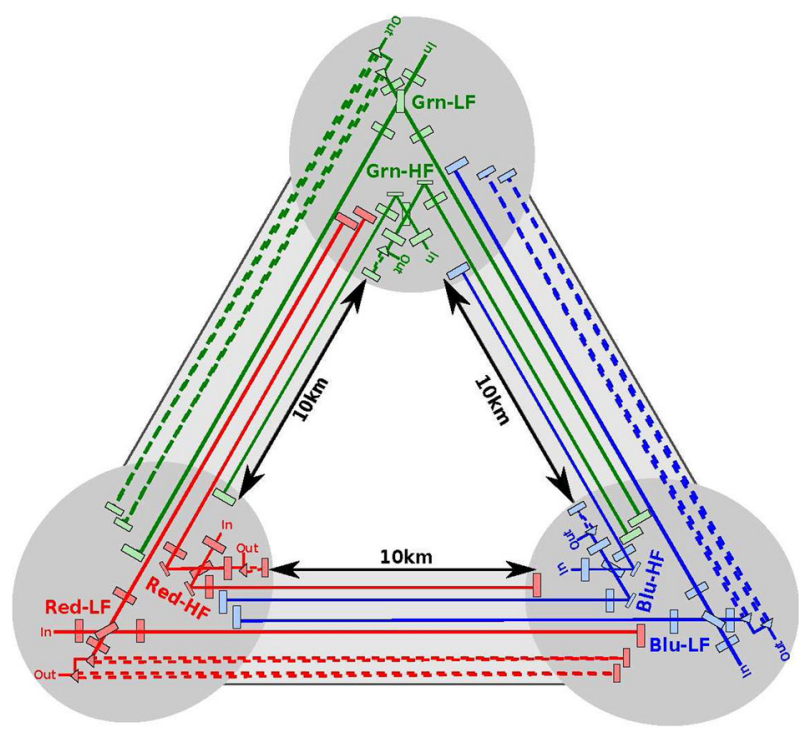

Fig. 1 Schematic Layout of the ET detector: each color represents a xylophone detector, composed an LF (Low-Frequency) and an HF (High-Frequency) interferometer. The continuous lines represent the interferometers' cavities, while the dashed lines represent the auxiliary filter cavities

the probability to have the contribution of $\mathrm{NN}$ below a certain threshold during the transient gravitational wave $(\mathrm{GW})$ signal.

In the present paper, after recalling the fundamental layout of the ET detector we motivate the choice of the time scale of our seismic noise analysis by considering the search of intermediate-mass black holes binaries. Hence, we describe and show the seismic noise in the Sos Enattos site, we estimate the NN induced in the ET detector and the probability distribution to not perturb the detection during the signal transient.

\section{ET optical layout}

The optical layout of the ET detector is reported in Fig. 1. It is composed by a total of six Michelson interferometers each lying on two sides of an equilateral triangle. The total observation bandwidth will extend from $2 \mathrm{~Hz}$ to several $\mathrm{kHz}$, obtained by using a xylophone configuration, i.e., two kinds of interferometers: the LF (Low Frequency), optimized to the low frequency part of the spectrum, and the HF (High Frequency) optimized for the intermediate and high part of the spectrum. The arms of each interferometer form an angle of 60 degrees, and each arm is actually a $10 \mathrm{~km}$ long Fabry-Perot cavity whose input mirror is placed close to the interferometer beam splitter. Multi-interferometer triangular configuration has been designed to improve, with a single detector, the sensitivity to the two GW polarizations. So the different outputs of each interferometer are used to reconstruct the direction and polarization of the gravitational wave [5]. 
A Newtonian noise glitch lasting several seconds is expected to enter in the three low-frequency interferometers of the ET detector, degrading the sensitivity of the whole detector. ${ }^{1}$ This can be more clearly appreciated by observing the optical layout of the ET detector, in particular by paying attention to the LF interferometers. Each corner station hosts the two input test masses of one interferometer and one end test mass belonging to each of the two other interferometers. So, each interferometer will have at least a cavity-test mass nearby a cavity mass of both the other interferometers. The distances among these masses, in the present design, will be of the order of one $\mathrm{km}$, so it is expected that all the three interferometers will suffer the Newtonian glitch. A deep NN correlation study has yet to be carried out. The present analysis follows the prescription in [17] where the noise projection on the whole ET sensitivity is obtained by considering the effect of $\mathrm{NN}$ on a single Detector, without accounting for the 60 angle, and considering the body waves polarization $p=1 / 3$. (see the section Newtonian "Noise estimation by Seismic Noise")

\section{Choice of time window}

The gravitational wave from a coalescing a binary system is the well tested "chirp" $[1,18]$, composed by three phases: inspiral, merger and ring down. During the inspiral phase, the amplitude and frequency of the wave smoothly rise until the beginning of the merger phase, a fast transient where the amplitude reaches its maximum and then decrease; once merged, in the ring down phase, the system relaxes loosing energy by oscillating in its quasi-normal modes, at higher frequencies and much lower amplitude.

Until now, the well-observed gravitational signals correspond to the latest cycles of the inspiral phase and to the merger, and we will focus on these signals to choose the time duration of the "glitch" in our seismic analysis. For the purpose of the present paper, we focus on the inspiral phase, because it produces a clear gravitational signal made of several cycles that allow the analysis to clearly distinguish it from other spurious burst. The upper frequency reached by the gravitational wave during the inspiral phase can be approximated as two times (to take into account quadrupolar radiation) the frequency $f_{I S C O}$ of the last stable orbit $[18,19]$ :

$$
f_{I S C O} \approx 2.2 \mathrm{kHz}\left(\frac{\mathrm{M}_{\odot}}{m}\right)
$$

where $\mathrm{M}_{\odot}$ is the solar mass, $m=m_{1}+m_{2}$ is the total mass of the binary system and $m_{1}$ and $m_{2}$ the masses of the two components. If we concentrate on the gravitational wave bandwidth of $2-10 \mathrm{~Hz}$, the maximum frequency $f_{\max }$ of the inspiral phase corresponds to binary systems of total mass of about $440 M_{\odot}$, in the detector frame.

The time to coalescence, $\tau$ for a binary systems is given by:

$$
\tau \approx 2.18 \mathrm{~s}\left(\frac{1.21 \mathrm{M}_{\odot}}{M_{c}}\right)^{5 / 3}\left(\frac{100 \mathrm{~Hz}}{f}\right)^{8 / 3}
$$

being $M_{c}$ the chirp mass:

$$
M_{c}=\frac{\left(m_{1} m_{2}\right)^{3 / 5}}{\left(m_{1}+m_{2}\right)^{1 / 5}}
$$

\footnotetext{
1 Actually it enters in all the six interferometer; we are interested to the three low-frequency interferometers because their better low-frequency sensitivity could be affected by NN. On the contrary, the high-frequency interferometers, having a worst sensitivity at low frequency, are not affected by NN.
} 
For a total mass $m=440 \mathrm{M}_{\odot}$ with equal single masses $m_{1}=m_{2}$, the chirp mass is $M_{c} \approx$ $200 \mathrm{M}_{\odot}$. If we consider the signal entering in the observation bandwidth at the frequency $f=2 \mathrm{~Hz}$, it will remain within the observation band, until its merging, for a time $\tau=16 \mathrm{~s}$. Less symmetric systems, with correspondingly smaller chirp mass, will stay longer in the detection bandwidth. As an example, a system with the same total mass but with a mass ratio of $m_{1} / m_{2} \approx 20$, as given by an intermediate black hole with $m_{1}=420 \mathrm{M}_{\odot}$ and a stellar black hole with $m_{2}=20 \mathrm{M}_{\odot}$, will end the inspiral phase at $10 \mathrm{~Hz}$, after having lived about $90 \mathrm{~s}$ in the bandwidth. In the same way, more massive systems will end the inspiral phase at lower frequencies and will remain in the bandwidth from tens of seconds to several minutes depending on the mass ratio.

Guided by these considerations, we choose a time duration of 1 minute to define a seismic time window, where a burst of seismic noise would be potentially invasive for the search of coalescing Intermediate Mass Black Hole binary systems.

\section{Newtonian noise estimation by seismic noise}

In underground detectors, the main sources of Newtonian noise are both surface and body waves. At present, the noise projections are still under study, to take into account the scattering from the cavern and from the inhomogeneity of the soils [13]. For what concerns the surface wave, it is presently expected that their contribution to $\mathrm{NN}$ is negligible if the detector is located underground at a depth of few hundreds meter [20], as in the case of the ET interferometer.

Under this condition, the main contribution to $\mathrm{NN}$ is expected to come from body waves $[17,20]$. In the rest of the paper, we will follow this assumption: we will estimate the Newtonian noise on the detector by the seismic noise measurements at $84 \mathrm{~m}$ depth and project it on the ET sensitivity assuming that it is entirely composed by body waves.

Body waves are described with the help of the seismic displacement vector $\boldsymbol{\xi}$ [13], which is a useful method because it can be measured directly:

$$
\xi^{P, S}(\mathbf{r}, t)=\mathbf{e}^{P, S} \xi_{0}^{P, S}\left(\mathbf{k}^{P, S}, \omega\right) \exp \left(i\left(\mathbf{k}^{P, S} \cdot \mathbf{r}-\omega t\right)\right)
$$

The indexes $P$ and $S$ distinguish the compressional $(P)$ and shear $(S)$ waves, respectively, $\mathbf{k}$ is the wave vector, $\mathbf{e}_{k}$ is the polarization unit vector, and $\omega$ is the angular frequency. Compressional waves are longitudinal: $\mathbf{e}^{P}=\mathbf{k}^{\mathbf{P}} / k^{P}$ while shear waves are transversal: $\mathbf{e}^{\mathrm{S}} \cdot k^{S}=0$. by:

Following the Newton law, the acceleration $\delta \mathbf{a}$ of a mass placed at the point $\mathbf{r}_{0}$ is given

$$
\delta \mathbf{a}\left(\mathbf{r}_{0}, t\right)=-G \int d V \rho(\mathbf{r})\left(\xi(\mathbf{r}, t) \cdot \nabla_{0}\right) \frac{\mathbf{r}-\mathbf{r}_{0}}{\left|\mathbf{r}-\mathbf{r}_{0}\right|^{3}}
$$

In the first approximation, the underground cavities of the detector can be assumed to be spherical, with radius much shorter that the seismic wavelengths and the mass in the center [13]. Furthermore, assuming that the density variations in space and time can be neglected, setting $\rho(\mathbf{r}, t)=\rho_{0}$ constant, the above integral can be solved, giving in the frequency domain:

$$
\delta \mathbf{a}(\omega)=\frac{4 \pi}{3} G \rho_{0}\left(2 \xi^{P}(\omega)-\xi^{S}(\omega)\right)
$$


The projection of the noise on the ET sensitivity is performed in the approximation that the NN contribution on the four test masses of the interferometer is not coherent [21]. The Power Spectral Density (PSD) of the measured seismic noise $S(\xi, \omega)$ can be expressed as the superposition of $P$ and $S$ contribution by defining a mixing parameter $p$ as the fraction of compressional contribution: $S\left(\xi^{P}, \omega\right)=p S(\xi, \omega)$ and $S\left(\xi^{S}, \omega\right)=(1-p) S(\xi, \omega)$. Following the discussion in [17], we assume that all three body-wave polarizations carry the same average displacement power and set $p=1 / 3$.

Under these hypothesis, the amplitude spectral density (ASD) of the Newtonian noise contribution to the detector output $h(t)_{N N}$ is given by:

$$
\tilde{h}_{N N}(f)=\frac{4 \pi}{3} G \rho_{0} \frac{2 \sqrt{2}}{L} \frac{1}{(2 \pi f)^{2}} \tilde{x}(f)
$$

where $f$ is the frequency, $\tilde{x}$ is the ASD of the measured seismic noise and $L$ the arm-length of each interferometer.

\section{Amplitude spectral density}

The measurement campaign used for this work follows a previous long data taking performed for more than one year in the Sos Enattos mine. The results of those measurements are reported in a previous paper and refer mostly to data collected by two seismometers placed at different depths. Both instruments are Nanometrics Trillium 240; the first (SOE1) is placed at the depth of $84 \mathrm{~m}$ and the second (SOE2) at $111 \mathrm{~m}$. Detailed description on seismometer installations, site geological characterization and median on seismic measurements can be found in [22].

The long-term analysis of the seismic data has proven that the Sos Enattos site is very quite in terms of seismic noise at depth. This leads to the need of reconfiguring the digitizer gain since the standard configuration was hitting its self-noise level in the frequency band 1-20 Hz. The present study uses data from the update configuration for the period August 1 - August 26, 2020.

The results mediated on the period are summarized in Fig. 2. The black curve is the design total noise of ET, that is expected to be limited by NN in the region from 2 to about $7 \mathrm{~Hz}$ [21]. The target Newtonian noise contribution in ET project is given by the dashed line. The red line is the median amplitude spectral density (ASD) of the Newtonian noise in Sos Enattos with the seismic noise being measured with SOE1 and projected on the sensitivity following Eq. 6 and assuming $\rho_{0}=2.7 \cdot 10^{3} \mathrm{~kg} / \mathrm{m}^{3}$.

It can be appreciated that the median is compatible with the ET Newtonian noise project in almost the whole range of frequency. In the lowest range of frequencies, between 2 and 3 $\mathrm{Hz}$, an active reduction of about a factor of two would be sufficient to bring the whole curve below the project curve.

These results are extremely encouraging, in particular because they are conservative: although not in operation, there are accesses to the mine for maintenance and environment protection and the ET detector will be located at higher depth with respect to SOE1.

In Fig. 3, the ASD median on the whole period is reported, together with the ASD median on the holiday period (August 8th-August 23rd) when the mine was closed and the median ASD during the nights of the whole period. It can be observed that during holidays there is a small reduction of the noise while during nights the noise level is remarkably lower than ET design in almost the whole bandwidth. 


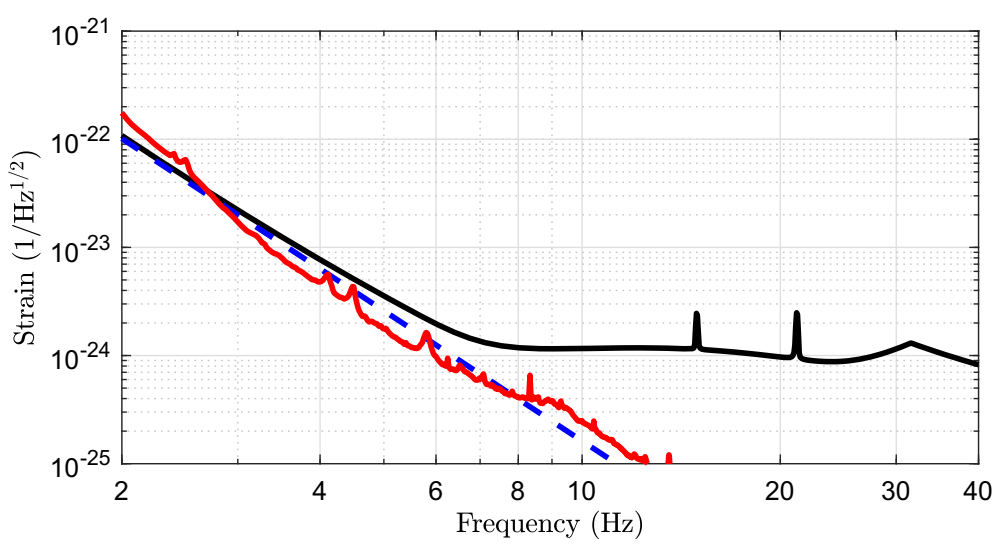

Fig. 2 ASD of ET target sensitivity (black) compared with target NN contribution (dashed blue) and projected NN noise based on the median ASD of the seismic noise measurement (red)

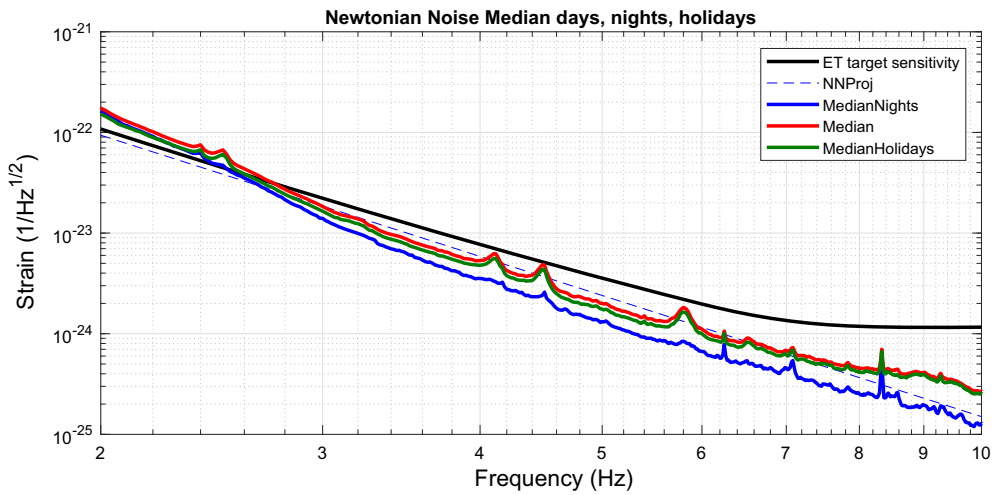

Fig. 3 ET target sensitivity and NN contribution zoomed in the bandwidth $2-10 \mathrm{~Hz}$

\section{Noise distribution}

The median of amplitude spectral density of NN is extremely encouraging. Nonetheless, the seismic noise is typically not stationary and its variability can be due both to long nightday variations and fast transients of anthropic or natural origin. We thus performed a first analysis on the distribution of the noise, to evaluate the probability to have the sensitivity of the detector degraded by Newtonian noise during the arrival of an IMBHs coalescence gravitational wave. Based on the considerations of the above sections, we focus our analysis in 1-minute windows and calculate the noise-to-target ratio (NTR) of the Newtonian noise with respect to the ET sensitivity defined as:

$$
\mathrm{NTR}=\sqrt{\frac{1}{\Delta f} \int d f \frac{\tilde{N} * \tilde{N}}{S_{h}}}
$$

$S_{h}$ is the PSD of the ET target sensitivity and $\tilde{N} * \tilde{N}$ is the PSD of the Newtonian noise. As usual, the normalization to the bandwidth assures that if the NN is equal to the ET target sensitivity the value NTR $=1$ is obtained. Considering that in $2-10 \mathrm{~Hz}$ bandwidth, NN is 


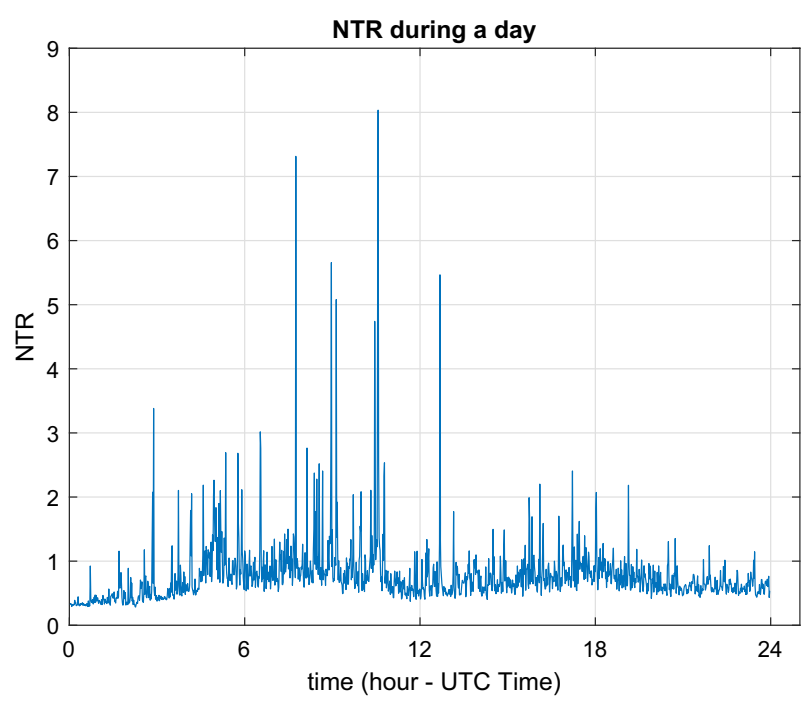

Fig. 4 Time evolution of the $2-10 \mathrm{~Hz}$ 1-minute NTR during a day. The time is UTC, the local time being UTC+2 hours

expected to limit $(2-7 \mathrm{~Hz})$ or contributing $(7-10 \mathrm{~Hz})$ to ET noise, unity can be taken as the critical value to assess if an excess of Newtonian noise, in the minute considered, is degrading the target sensitivity.

The time variability of seismic noise can be appreciated, for example in Fig. 4, where it is reported the NTR value, in 1-minute windows, in the bandwidth $2-10 \mathrm{~Hz}$, evaluated in each minute for a whole day. The floor follows a night-day variation, and fast transients are more frequent during the morning.

In Fig. 5, it is reported the same NTR value, evaluated in each minute for a whole day, in the different days. The time axis is UTC, so the local time is 2 hours more than what reported in the time axis. It can be appreciated that the noise events appear early in the morning, around 4 to 5 o'clock local time and last until late early afternoon. During nights, the noise events reduce drastically. Also it can be appreciated that during the second part of the month, corresponding to holidays, the events are less with respect to the first days. This behavior is consistent with a prevalent anthropic local excitation of the site, that will be further investigated.

The results with respect to degradation of ET sensitivity are reported in Fig. 6 for the whole period. The distribution is picked at a value lower than unity. The corresponding probability of having a NTR lower than unity is $P($ NTR $<1)=0.60$ in agreement with the value of the median shown in Fig. 2. The probability of having NTR $<1.5$, thus admitting a small degree of degradation, is about 0.8 . If the noise reduction of a factor 2 in the $2-3 \mathrm{~Hz}$ band is taken into account (see [20]), the NTR probability of being better than unity increases to about $97 \%$.

These values are extremely encouraging, in particular, if we take into account that human activities still occur into the mine. This can result in an overestimation of the intrinsic noise of the site.

To further remark this point, in Fig. 7 we report the NTR distribution during the nights and in Fig. 8 the NTR distribution during the two summer holidays weeks. 


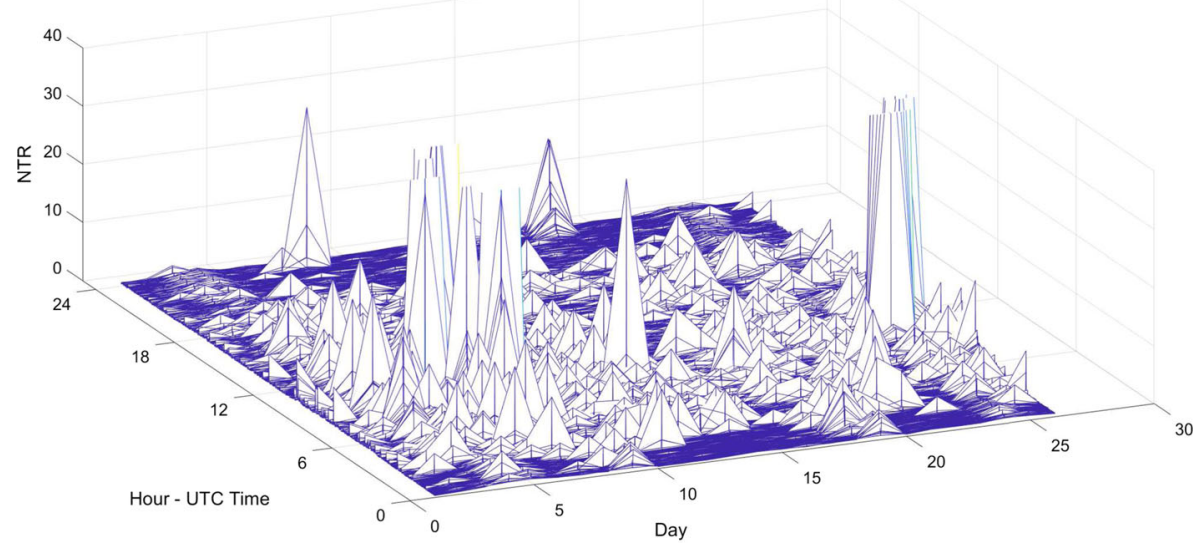

Fig. 5 Time evolution of the $2-10 \mathrm{~Hz} 1$-minute NTR during the various days

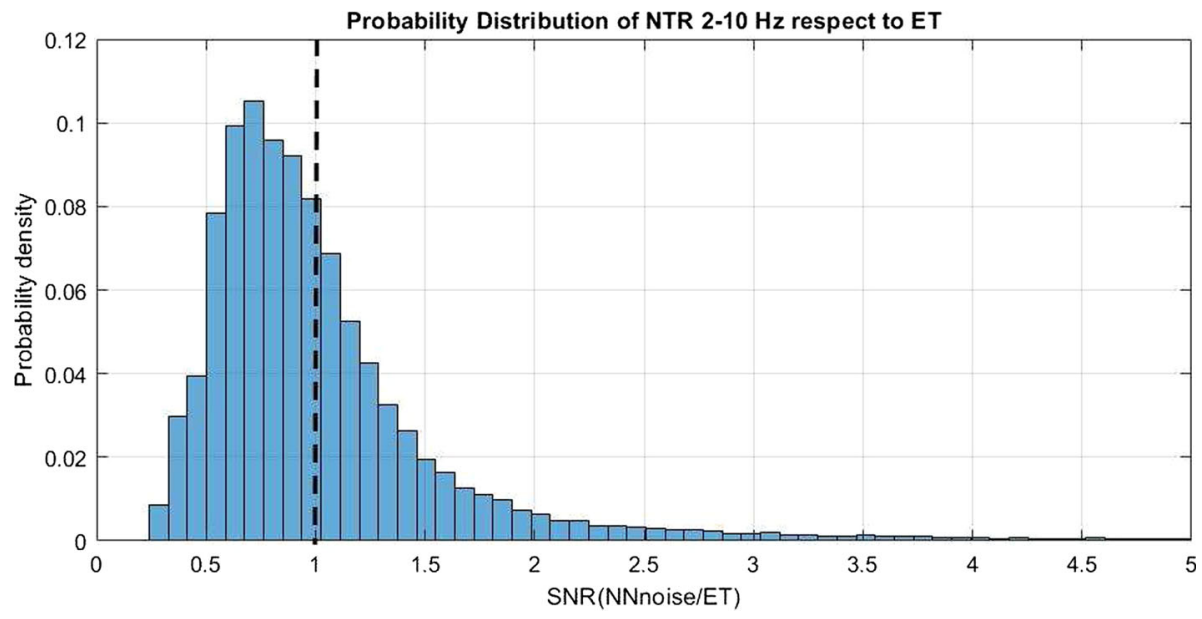

Fig. 6 NTR probability distribution over the whole period

During night, the probability to be better than unity is $86 \%$ without any reduction and increases to $95 \%$ if a slight degradation to NTR up to 1.5 is considered. Finally, when the 16 days of holidays are considered, from August 8th to August 23rd, the probability to be better than unity becomes $76 \%$. Let us remark that also in this condition the present results are conservative. Indeed the mine, even in absence of environmental works, is still operated with maintenance bilge pumps, guardian's movements and free to be accessed up to the entrance by sheep and tourists. 


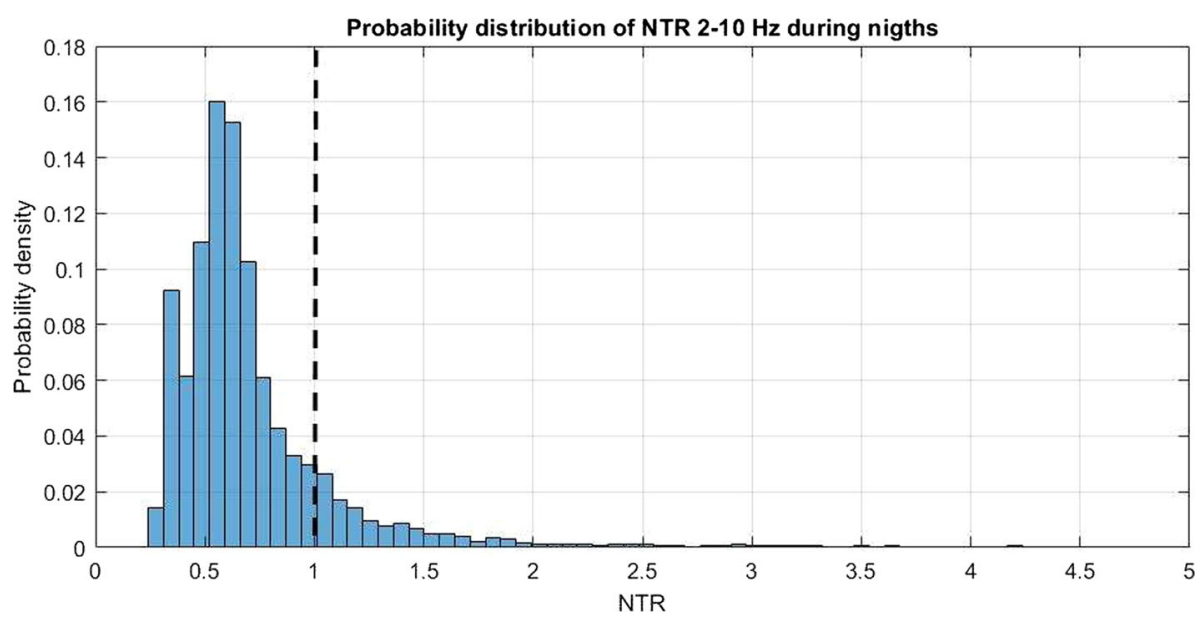

Fig. 7 NTR probability distribution during the nights

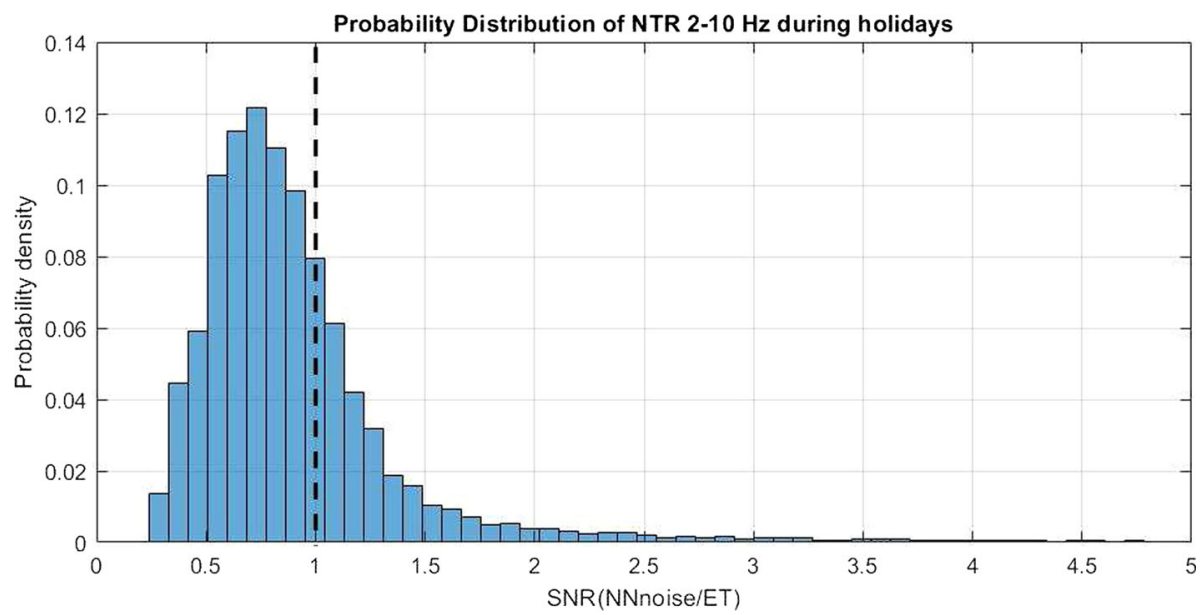

Fig. 8 NTR probability distribution during the holidays weeks

\section{Interferometer L shaped}

The classical L-shape interferometer is still an option for ET or for other third-generation detectors. If operated in a network, this would warrant a very efficient detection of the direction and the polarization of the GW sources, with a simpler realization.

In the following, we analyze the NTR assuming a single xylophone interferometer, composed of $20 \mathrm{~km}$ long arms , with target sensitivity equal to the one planned for ET. The results, referred to the whole data set, are reported in Fig. 9. The probability that the Newtonian noise degrades the sensitivity is about $5 \%$, compatible with the typical duty cycle of gravitational wave detectors. When this configuration is considered, very likely the NN reduction would not be necessary. 
Fig. 9 NTR probability distribution during the period for Sos Enattos (blue) for a $20 \mathrm{~km}$ L-shaped detector

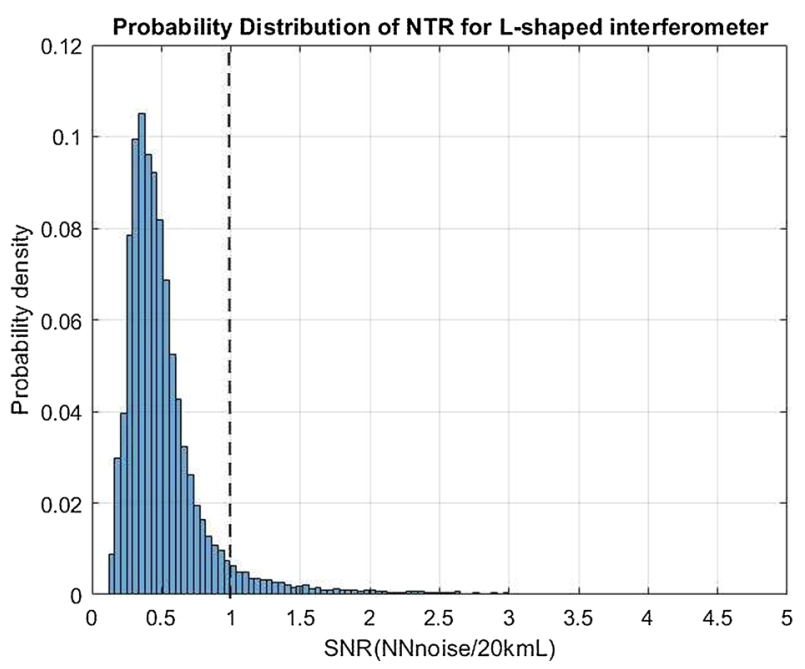

\section{Discussion}

From this preliminary analysis, it results that the ET detector, if hosted by the Sos Enattos site, will be for the great majority of time in the position of detecting the new sources like IMBH with no degradation of sensitivity even not considering any reduction of the Newtonian noise. These results, whenever showing an optimal site for hosting the detector, can be considered as preliminary for three main reasons. The first is that the noise projection of Eq. 6 is an approximation that does not consider in detail the coherence among the masses of each interferometer and among the signals of the three LF interferometers. Second, the mine is still maintained, various activities are performed in the immediate neighborhood, and the seismometers are not yet placed at their ultimate depth of $250 \mathrm{~m}$. In these sense, the presented results can be considered as upper limits in the noise, as it can be particularly appreciated by looking at the behavior during nights and holidays. Part of the next activity will be the better isolation of the seismometers and, if needed, an evaluation of the seismic noise of the site with boreholes at $250 \mathrm{~m}$ depth. Third, a study aimed to locate the different sources of seismic noise is undergoing, with the aim also to help in the design of low self-noise generating ET infrastructures. Finally, the case of single $20 \mathrm{~km}$ arm L-shaped xylophone interferometer has been considered. The Newtonian noise is not degrading the sensitivity for more that about $5 \%$ of time, which implies that no Newtonian noise reduction would be needed in this case.

Acknowledgements This work has been supported by: INFN with Protocollo di Intesa tra Ministero dell'Universita' e della Ricerca, la Regione Autonoma della Sardegna, l'Istituto Nazionale di Fisica Nucleare e l'Università degli Studi di Sassari; University of Sassari with Accordo di Programma tra la Regione Autonoma della Sardegna, l'Università degli Studi di Sassari, l'Istituto Nazionale di Fisica Nucleare, l'Istituto Nazionale di Geofisica e Vulcanologia, l’Università degli Studi di Cagliari e l'IGEA S.p.a (progetto SAR-GRAV, funds FSC 2014-2020 Patto per lo sviluppo della Regione Sardegna); COST Action CA17137; Sapienza University of Rome "Fondi di Ateneo 2017; Università degli Studi di Sassari with "Fondo di Ateneo per la ricerca 2019" ; Istituto Nazionale di Fisica Nucleare and by the Italian Ministero dell' Università e della Ricerca with PRIN 2017 Research Program Framework, n. 2017SYRTCN. The authors thanks L. Loddo and IGEA miners for their support during operation in Sos Enattos.

Funding Open access funding provided by Università degli Studi di Napoli Federico II within the CRUICARE Agreement. 
Data Availability Statement This manuscript has associated data in a data repository. [Authors' comment The datasets generated and analysed during the current study are available from the corresponding author on reasonable request.]

Open Access This article is licensed under a Creative Commons Attribution 4.0 International License, which permits use, sharing, adaptation, distribution and reproduction in any medium or format, as long as you give appropriate credit to the original author(s) and the source, provide a link to the Creative Commons licence, and indicate if changes were made. The images or other third party material in this article are included in the article's Creative Commons licence, unless indicated otherwise in a credit line to the material. If material is not included in the article's Creative Commons licence and your intended use is not permitted by statutory regulation or exceeds the permitted use, you will need to obtain permission directly from the copyright holder. To view a copy of this licence, visit http://creativecommons.org/licenses/by/4.0/.

\section{References}

1. B.P. Abbot et al., Observation of Gravitational Waves from a Binary Black Hole Merger. Phys. Rev. Lett. 116, 061102 (2016)

2. B.P. Abbot et al., GW170817: Observation of Gravitational Waves from a Binary Neutron Star Inspiral. Phys. Rev. Lett. 119, 161101 (2017)

3. B. P. Abbot et al. (2019) GWTC-1: A Gravitational-Wave Transient Catalog of Compact Binary Mergers Observed by LIGO and Virgo during the First and Second Observing Runs, Phys Rev X, 9: 031040

4. D. Reitze et al. (2019) Cosmic Explorer: The US CoNTRibution to Gravitational-Wave Astronomy beyond LIGO, Bull. Am. Astron. Soc., 51: 51

5. M. Punturo et al., The Einstein Telescope: A third-generation gravitational wave observatory. Class. Quantum Grav. 27, 194002 (2010)

6. B. Sathyaprakash et al., Scientific Objectives of Einstein Telescope, Class. Quant. Grav, 29, (2012) 124013 and Class. Quant. Grav., 30, (2013) 079501 (erratum)

7. M. Mezcua, Observational evidence for intermediate-mass black holes. Int. J. Mod. Phys. D 26, 1730021 (2017)

8. B.P. Abbot et al., GW190521: A Binary Black Hole Merger with a Total Mass of 150M. Phys. Rev. Lett. 125, 101102 (2020)

9. B. P. Abbot et al., (2020) Properties and Astrophysical Implications of the 150 M Binary Black Hole Merger GW190521, Astrophys J Lett, 900(1): L13

10. B.P. Abbot et al., Characterization of transient noise in Advanced LIGO relevant to gravitational wave signal GW150914. Class. Quantum Grav. 33, 134001 (2016)

11. I. Fiori et al., The hunt for environmental noise in virgo during the third observing run. Galaxies $\mathbf{8}, 82$ (2020)

12. T. Accadia et al., Noise from scattered light in Virgo's second science run data. Class. Quantum Grav. 27, $194011(2010)$

13. J. Harms, Terrestrial gravity fluctuations. Living Reviews in Relativity 22, 6 (2019)

14. S. Koley et al, First results of seismic studies of the Belgian-Dutch-German site for Einstein Telescope, NIKHEF public report, https://www.nikhef.nl/wp-content/uploads/2019/10/Terziet-Drilling-CampaignFinal-NoC.pdf

15. L. Naticchioni et al., (2014) Microseismic studies of an underground site for a new interferometric gravitational wave detector, Class. Quantum Grav., 31:105016

16. L. Naticchioni et al., Characterization of the Sos Enattos site for the Einstein Telescope. J. Phys. Conf. Ser. 1468, 012242 (2020)

17. F. Amann et al. (2020) Site-selection criteria for the Einstein Telescope, Rev. Sci. Instrum. $91: 9$

18. B. P. Abbot et al., Tests of general relativity with GW150914 Phys. Rev. Lett., 116, (2016) 221101, and Phys. Rev. Lett., 121, (2018) 129902 (erratum)

19. M. Maggiore (2008) Gravitational Waves vol. 1 Theory and Experiments, Oxford University Press, Oxford

20. F. Badaracco, J. Harms, Optimization of seismometer arrays for the cancellation of Newtonian noise from seismic body waves. Class. Quantum Grav. 36, 145006 (2019)

21. S. Hild et al., Sensitivity studies for third-generation gravitational wave observatories. Class. Quantum Grav. 28, 094013 (2011)

22. M. Di Giovanni et al., A seismological study of the Sos Enattos area, the Sardinia candidate site for the Einstein Telescope. Seismol. Res. Lett. 92, 352-364 (2021) 